\title{
A Design Proposal for Ultrawide Band Frequency Selective Surface
}

\author{
Francisco Carlos Gurgel da Silva Segundo, Antonio Luiz Pereira de Siqueira Campos, \\ Communication Engineering Department, Federal University of Rio Grande do Norte, Av. Senador Salgado \\ Filho, 3000, Lagoa Nova, Natal, RN, Brazil, CEP: 59072-970, Tel./fax: +55-21-84-3215-37, e-mail: \\ segundoengeletrica@gmail.com,antonio.luiz@pq.cnpq.br \\ Alfredo Gomes Neto \\ Federal Institute of Education, Science and Technology of Paraíba, Campus João Pessoa, Av. 1 de maio, 720, \\ Jaguaribe, João Pessoa, PB, Brazil, CEP 58015-430, alfredogomesjpa@gmail.com
}

\begin{abstract}
In this paper a three layer FSS is described which provides a ultrawide band (UWB) response. The layers use plain rectangular patches, and rectangular patches with one or two notches which provide multi-band responses; by cascading them an UWB response is obtained covering the band $4.05-14.12 \mathrm{GHz}$, for vertical polarization and the band 5.05 $-15.00 \mathrm{GHz}$, for horizontal polarization. Transmission properties of the proposed structure were simulated and measured.
\end{abstract}

Index Terms - FSS, Rectangular Patch Element, Simple Modification, Cascaded Structure, Ultrawideband Response.

\section{INTRODUCTION}

A frequency selective surface can be built as a two-dimensional periodic array of metallic patch elements acting as a filter and exhibiting a spectral selectivity that depends on the polarization of the incident wave, the geometry of the planar circuit and the spacing between the elements within the FSS structure, the substrate thickness and permittivity (Fig. 1) [1].

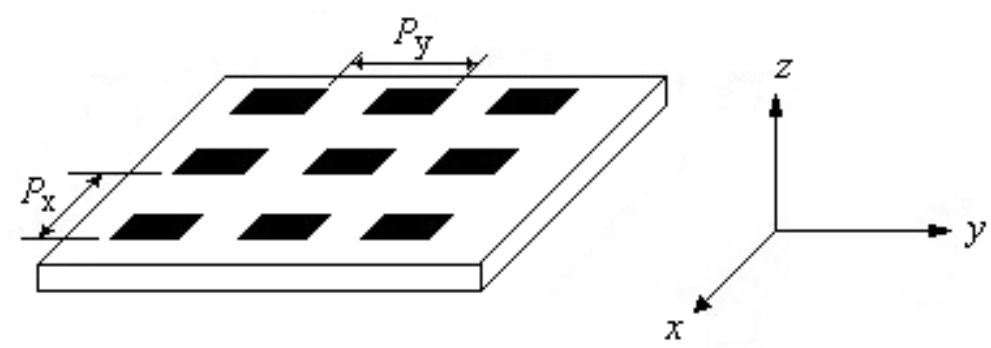

Fig. 1. FSS geometry.

Recent researches show that frequency selective surfaces (FSS) can be used to improve the response of antennas, spatial filters and absorbers. Additionally, the FSS can provide broadband characteristics to these structures, which is desirable in some applications [2] - [5].

In [2], Kazemzadeh and Karlsson proposed a design of an ultrawideband (UWB) absorber operating for a large range of incident angles and different polarizations. The authors designed a three layered 
capacitive absorber with an ultrawide bandwidth of $26 \mathrm{GHz}$, but the response frequency and the technique analysis was validated with another technique analysis. None experimental results were presented. In this design the authors used three dielectric layers.

Ranga et al. in [3] also use a multilayer FSS optimized to improve the gain of a UWB antenna. The design uses four FSS with square loop and double square loop arrays. The antenna is a slot antenna which yields an ultrawideband width of $140 \%(2.9 \mathrm{GHz}-18.38 \mathrm{GHz})$. With the FSS, the gain variation is $2 \mathrm{~dB}$ over the impedance bandwidth. As in [2], the authors in [3] used multilayer FSS to obtain the desired response.

In [4], the authors proposed a wideband FSS combining different elements. This design proposal exploits the possibilities to achieve UWB response using an array composed by the association of two patch elements per cell. A unified approach to design a UWB antenna reflector was presented. The use of multilayer FSS reflector increases the gain over the entire frequency band. The proposed FSS when coupled with a UWB antenna has a predicted impedance bandwidth of $133 \%$. As in [2], none experimental results were presented.

Pasian et al. in [5] use a FSS to increase the efficiency and bandwidth in UWB antenna arrays. To extend the frequency range of usability, an FSS was sandwiched between the antenna and the ground plane, providing an additional reflecting plane for a higher frequency band. The proposed backing structure composed by the FSS and the ground plane was designed to be used in conjunction with an UWB array of connected dipole antennas.

To enhance these applications, we found in literature, novel proposed FSS geometries with wide stop band characteristics [6] - [8]. In [6] the proposed design is compact and shows angular and polarization independency. The FSS proposed provides a wide stop band of $4.2 \mathrm{GHz}$ at $-10 \mathrm{~dB}$ reference level of insertion loss with its simple and easy to fabricate geometry. A single layer FSS was used in this design. Li et al. in [7] proposed a two layer FSS with complex geometries to obtain a wideband response. The bandwidth obtained was $4.22 \mathrm{GHz}-6.98 \mathrm{GHz}(51 \%)$. In [8] the authors present a new proposal for design of wideband FSS. The structure consists of an array composed by the association of two patch elements per cell: a square loop and a crossed dipole. The geometry was called crossed loop and it increases the bandwidth of the square loop and the crossed dipole, when analyzed separately. The structure obtained a bandwidth of $8.08 \mathrm{GHz}$, in the best case (59\%).

In [9] a single-layer, low-profile FSS with cross-dipole and ring elements has been presented. This FSS-based reflector demonstrates a very wide stop band from $6.5 \mathrm{GHz}$ to $14 \mathrm{GHz}$, with linearly decreasing reflection phase. So, this FSS is suitable for as an FSS-based reflector for printed antennas in low-profile wireless devices.

In this paper, we propose a combination of multi-narrow-band FSS geometries, presented in [10], to form a multilayered FSS for a very wide stop band response. The multi-narrow-band FSS are composed of rectangular patches and rectangular patches with notches. The resulting structure is a three multilayer FSS. This structure uses geometries much simpler than other solutions found in 
literature, being easy to manufacture. The band obtained was $11.10 \mathrm{GHz}(2.85 \mathrm{GHz}-13.95 \mathrm{GHz})$, bigger than other proposals in literature listed in this paper. Numerical and experimental results are compared and a very good agreement was obtained.

\section{FSS STRUCTURES AND SIMULATIONS}

In [10], the authors show that when notches are applied in rectangular patch, the FSS frequency response can be drastically affected. The frequency of resonance can be reduced and new resonances can be obtained. Furthermore, the bandwidth between resonances can be modified. Then, these characteristics can be used to design FSS with multiband response. The FSS with rectangular patch and rectangular patch with notches were designed and printed on a FR4 substrate, that is a low cost fiberglass, with dielectric constant $\varepsilon_{\mathrm{r}}=4.4$, thickness $h=1.6 \mathrm{~mm}$, and loss tangent $\delta=0.02$. Fig. 2 shows the geometries proposed for the patch elements with periodicities $P_{\mathrm{x}}$ and $P_{\mathrm{y}}$, that are $16 \mathrm{~mm}$ and $20 \mathrm{~mm}$, respectively. Next, a rectangular notch with dimensions of $2 \mathrm{~mm} \times 3 \mathrm{~mm}$ was applied to the patch in different positions, as illustrated in Fig. 2(b) and (c). The notches were inserted at $2 \mathrm{~mm}$ from the top of the patch element. A normal incidence was considered in the analysis.

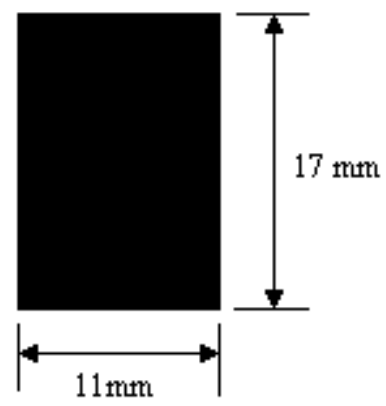

(a)

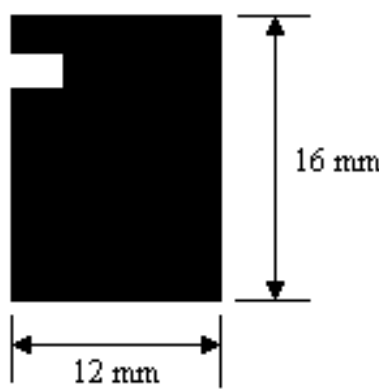

(b)

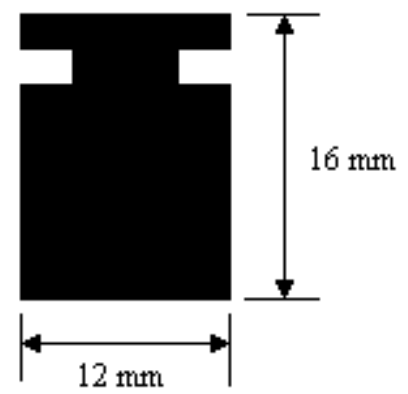

(c)

Fig. 2. Proposed geometries in [10]: (a) original rectangular patch element, (b) patch with one notch and (c) patch with two notches.

The idea is to cascade the three FSS with the geometries shown in Figure 2, to obtain an UWB response, as we can see in Fig. 3. The gaps between the three layers were equal to $10 \mathrm{~mm}$. We chose these gaps because when the distance between two layers is larger than half wavelength the frequency 
response of individual structures overlap, causing the desired effect of the UWB response.

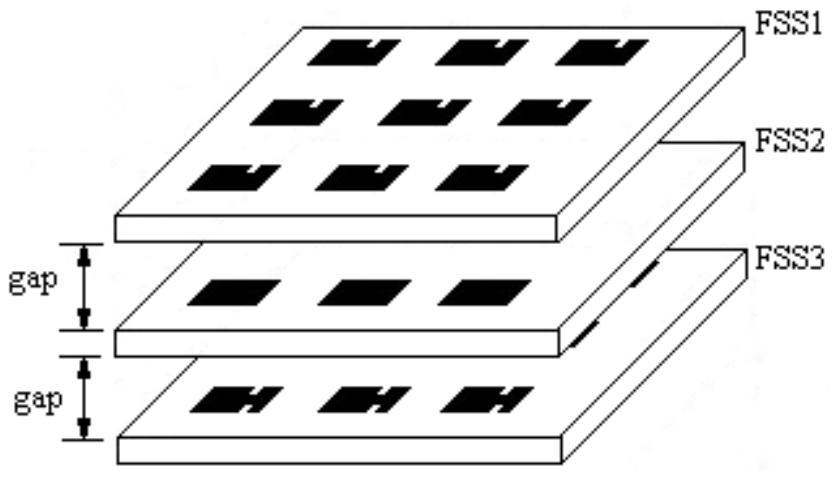

Fig. 3. A three layer FSS.

A numerical analysis of transmission properties of the designed FSS was performed through simulations using Ansoft Designer ${ }^{\mathrm{TM}}$ commercial software. In the analysis, a vertical polarization was considered for the electric field and a band of simulation was $2 \mathrm{GHz}-14 \mathrm{GHz}$, because this is the range of our measurement setup and it contains the UWB range. To compute the bandwidth, the level of $-10 \mathrm{~dB}$ was considered.

Fig. 4 illustrates the frequency responses of the transmission coefficient for the three structures individuals. The frequency response of the FSS 1, which uses the geometry shown in Fig. 2(b), exhibited four resonance bands with resonance frequencies. The FSS 2 uses a conventional rectangular patch as unit cell element as shown in Fig. 2(a). In this case, a unique resonance band occurs. The frequency response of the FSS 3, which uses the geometry shown in Fig. 2(c), exhibited two resonance bands.

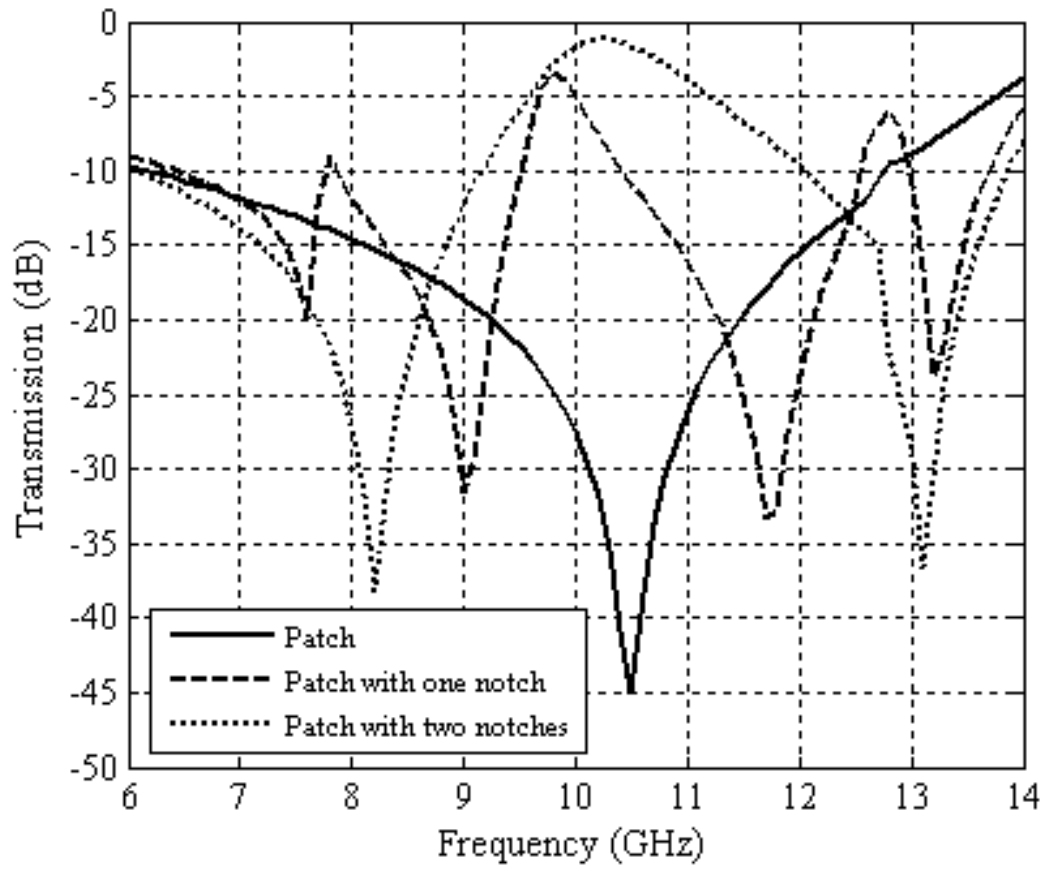

Fig. 4. Single layer FSS frequency responses. 
These resonance bands are listed in Table I. The idea is to cascade the three FSS, combining the resonance bands of each structure, to obtain a UWB response.

TABLE I. RESONANCE FREQUENCIES AND BANDWIDTHS FOR INDIVIDUAL FSS

\begin{tabular}{cccccc}
\hline \multicolumn{2}{c}{ FSS1 } & \multicolumn{2}{c}{ FSS2 } & \multicolumn{2}{c}{ FSS3 } \\
\hline $\boldsymbol{f}_{\mathbf{R}}(\mathbf{G H z})$ & $\mathbf{B W}(\mathbf{G H z})$ & $\boldsymbol{f}_{\mathbf{R}}(\mathbf{G H z})$ & $\mathbf{B W}(\mathbf{G H z})$ & $\boldsymbol{f}_{\mathbf{R}}(\mathbf{G H z})$ & $\mathbf{B W}(\mathbf{G H z})$ \\
7.60 (band 1) & 1.35 (band 1) & 10.50 (band 1) & 6.50 (band 1) & 8.20 (band 1) & 3.05 (band 1) \\
9.00 (band 2) & 1.65 (band 2) & - & - & 13.10 (band 2) & 1.80 (band 2) \\
11.70 (band 3) & 2.15 (band 3) & - & - & - & - \\
13.20 (band 4) & 0,70 (band 4) & - & - & - & - \\
\hline
\end{tabular}

\section{EXPERIMENTAL RESULTS AND DISCUSSIONS}

To validate our analysis, the three FSS prototypes were built and experimental characterizations were performed. So, we can compare our simulated results with measurements. Measured results were accomplished with the use of a Rohde \& Schwarz vector network analyzer (ZVB-14), with range of $10 \mathrm{MHz}-14 \mathrm{GHz}$, and two horn antennas operating in the range of $700 \mathrm{MHz}-18 \mathrm{GHz}$ with $16 \mathrm{dBi}$ of gain. The experimental results were not exhibit in [10]. A photograph of the front view setup measurement is shown in Fig. 5. The support which the FSS are fixed is circulated by absorbers to avoid diffraction at the edges.

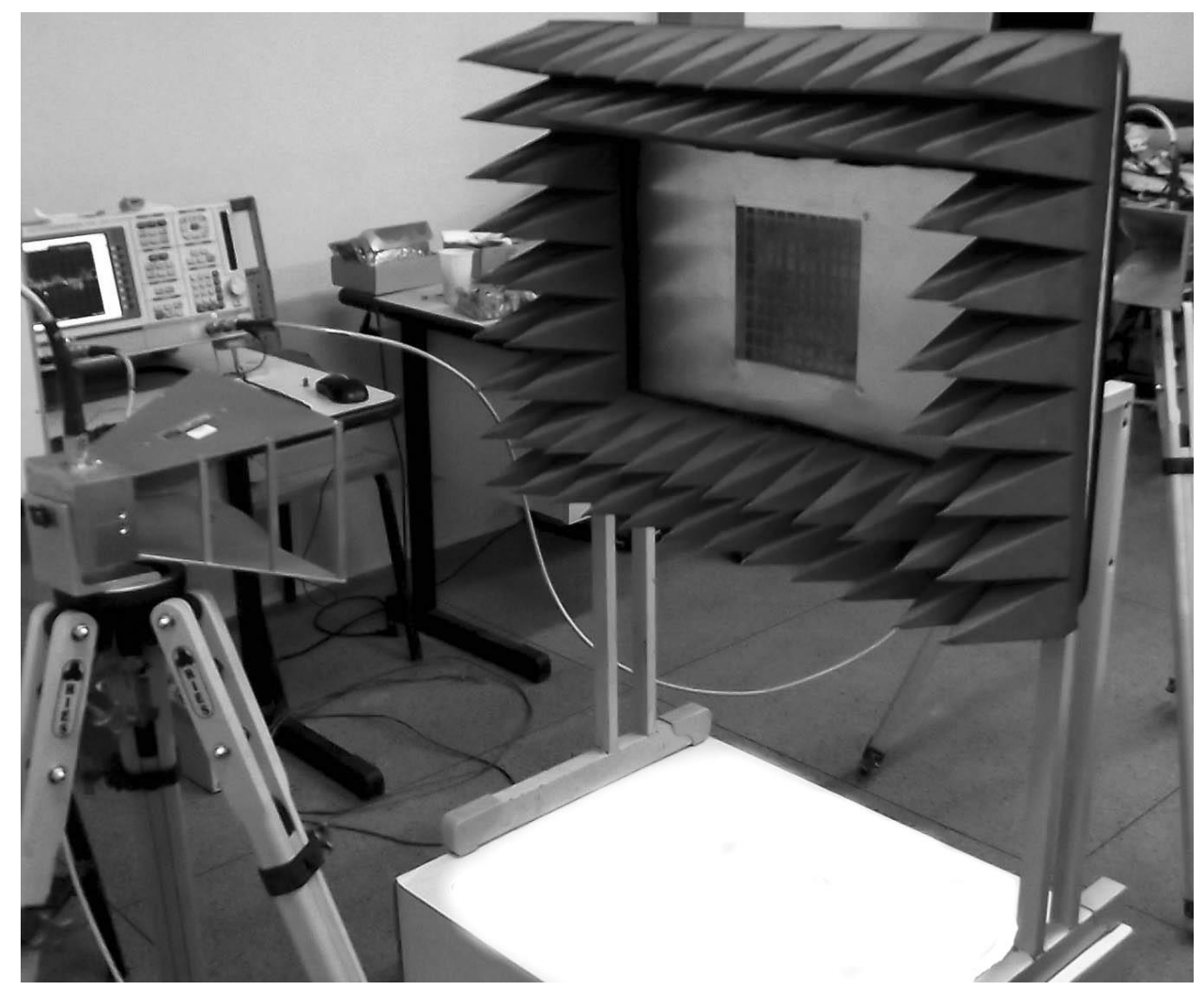

Fig. 5. Front view of the measurement setup.

Fig. 6 shows a back view of the support with the FSS fixed and a detail of what the FSS are spaced. We used spacers of Teflon with $5 \mathrm{~mm}$ of height. Two spacers were used between each FSS. Fig. 7 shows a photograph of the fabricated FSS and the Teflon spacers. 


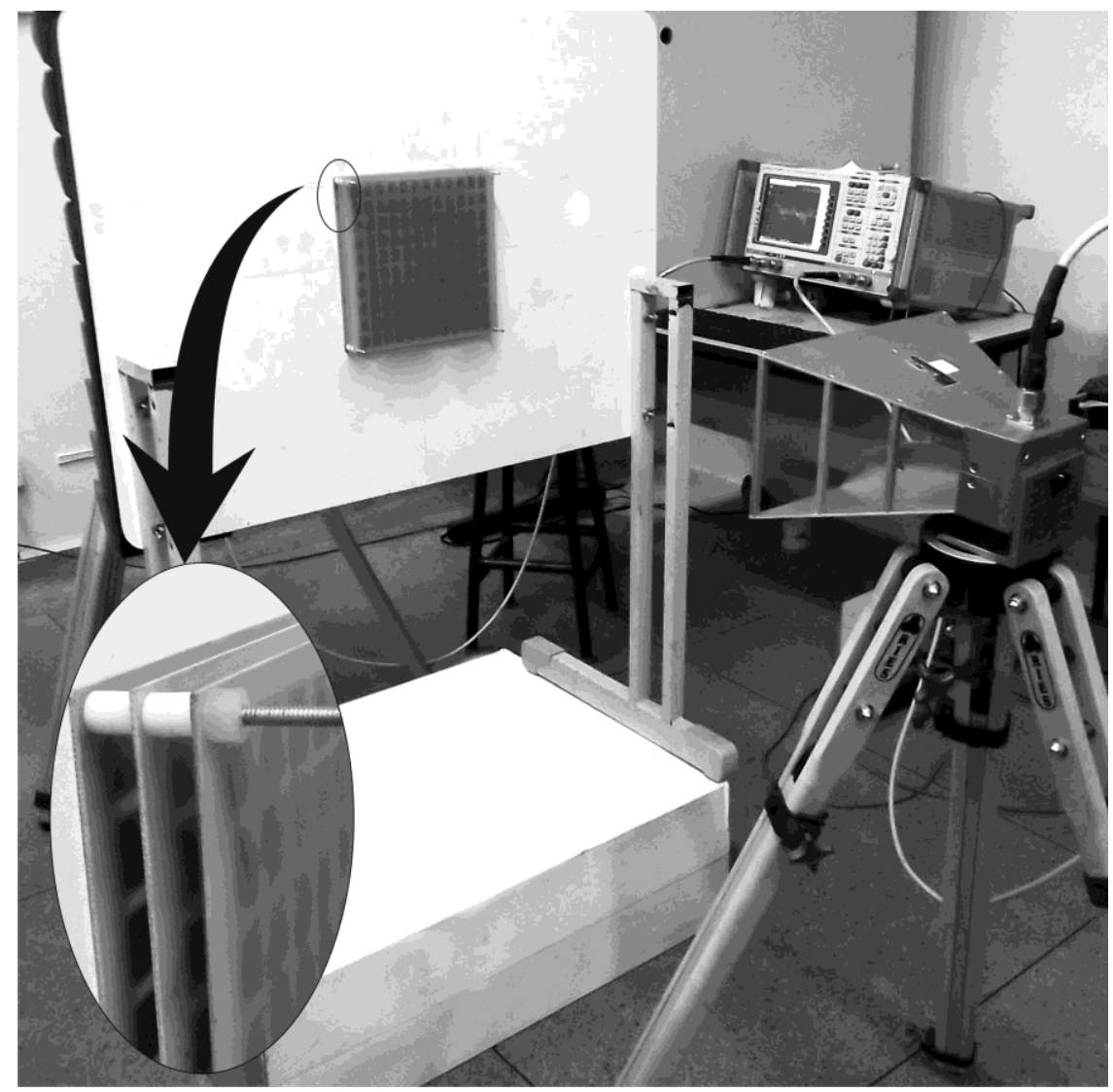

Fig. 6. Back view of the measurement setup and a detail of gaps between FSS.

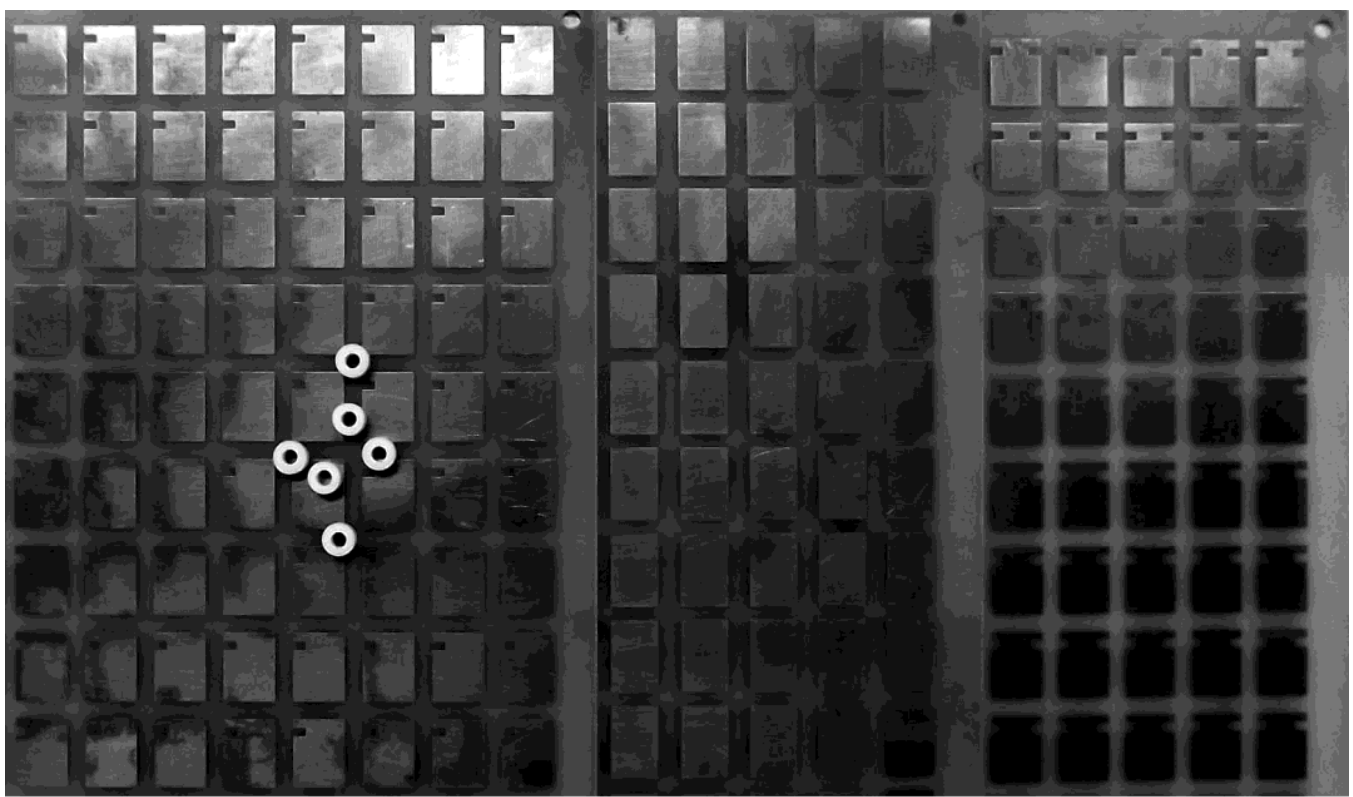

Fig. 7. Photograph of the fabricated FSS and Teflon spacers.

Fig. 8 shows a comparison between numerical and measured results for FSS1 using a conventional rectangular patch with one notch as cell element. Measured results present three resonant bands, because the first resonance also appears in measured results, but considering the $-10 \mathrm{~dB}$ level the first 
and second bands behave as a single band with bandwidth equal to $2.61 \mathrm{GHz}$. Also in this case, a good agreement between results is observed.

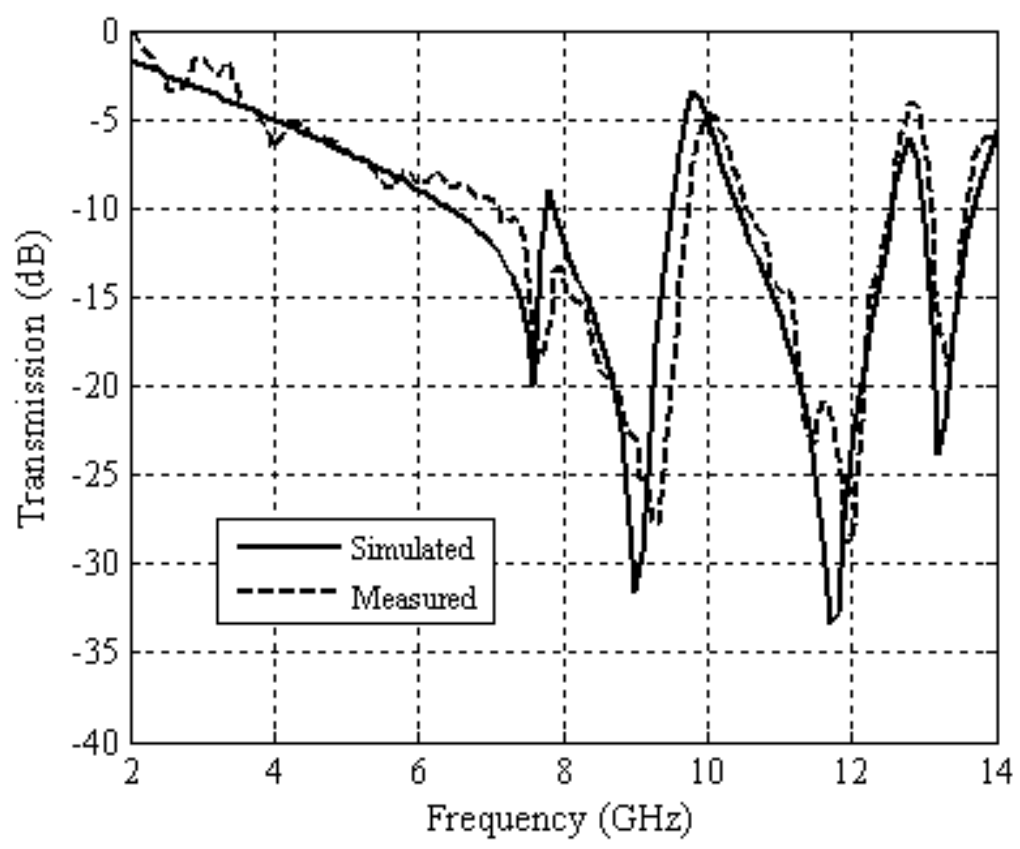

Fig. 8. Comparison between numerical and measured results for FSS1.

Fig. 9 shows a comparison between numerical and measured results for FSS2 using a conventional rectangular patch as a cell element. Measured results present a resonance frequency equal to 10.58 $\mathrm{GHz}$ and our simulated results present a resonance frequency equal to $10.50 \mathrm{GHz}$. The measured bandwidth was equal to $5.10 \mathrm{GHz}$ while simulated results present a bandwidth of $6.50 \mathrm{GHz}$. A good agreement between results is observed.

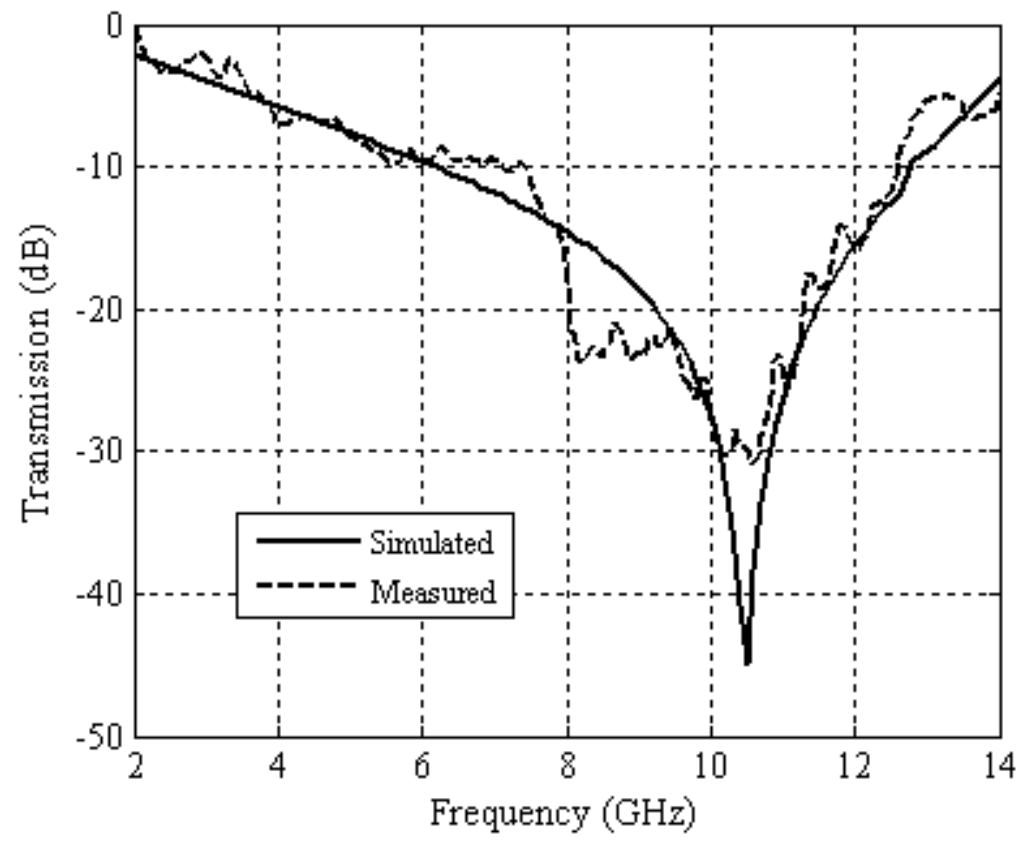

Fig. 9. Comparison between numerical and measured results for FSS2. 
Fig. 10 shows a comparison between numerical and measured results for the FSS3. Measured and simulated results exhibit two resonant bands and a good agreement between results is observed.

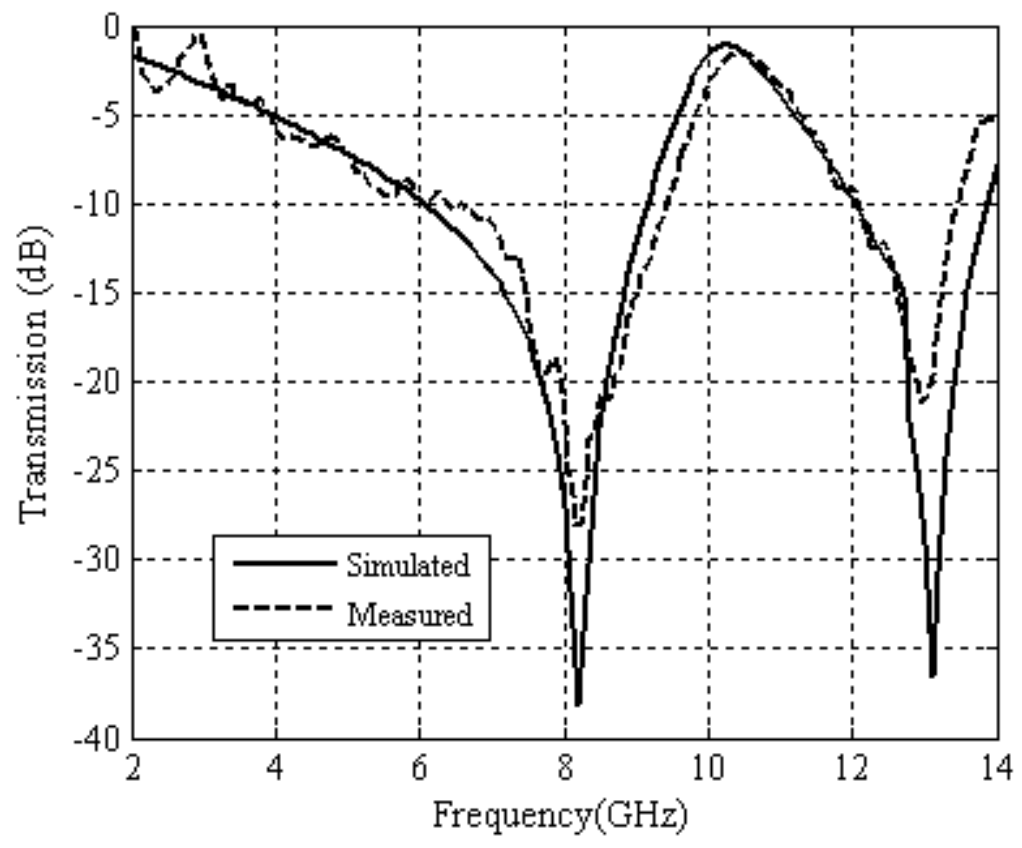

Fig. 10. Comparison between numerical and measured results for FSS3.

Based on the above analysis, a cascaded FSS structure is simulated using Ansoft Designer software and the corresponding simulation results are shown in Fig. 11. The transmission coefficient curves of FSS composite structure versus frequency for $0^{\circ} \sim 45^{\circ}$ angle of incidence and both polarization (vertical and horizontal) wave are shown in Fig. 11(a) and 11(b). It was seen in Fig. 11 that the stopband bandwidth of the FSS structure exhibits angular stability for vertical polarization maintaining the stopband bandwidth equal to $10.30 \mathrm{GHz}(4.05 \mathrm{GHz}-14.35 \mathrm{GHz})$. For horizontal polarization the FSS structure exhibits angular stability until $\theta=15^{\circ}$. Above this value the stopband bandwidth decreases to $4.5 \mathrm{GHz}$, what still is a very wide bandwidth, but shows that for horizontal polarization the cascaded FSS do not exhibit angular stability.

To demonstrate the accuracy of simulation results, experiment sample is fabricated. The measured results about transmission curves for both polarizations are shown in Fig. 12 and Fig. 13. In Fig. 12 we have a comparison between numerical and measured results for a three layer FSS cascading FSS1, FSS2 and FSS3. The simulated frequency response of the three overlapped FSS exhibit a bandwidth of $10.12 \mathrm{GHz}(4.00 \mathrm{GHz}-14.12 \mathrm{GHz})$. We can note that exist a difference of amplitude from $5 \mathrm{GHz}$ to $11 \mathrm{GHz}$. However, we are interested in the $-10 \mathrm{~dB}$ level and, bellow of this reference level, these differences are not relevant. A vertical polarization was considered in these results.

In Fig. 13 we done the comparison made in Fig. 12, but in this case, we consider a horizontal polarization. The simulated frequency response of the three overlapped FSS exhibit a bandwidth of $9.95 \mathrm{GHz}(5.05 \mathrm{GHz}-15.00 \mathrm{GHz})$. 


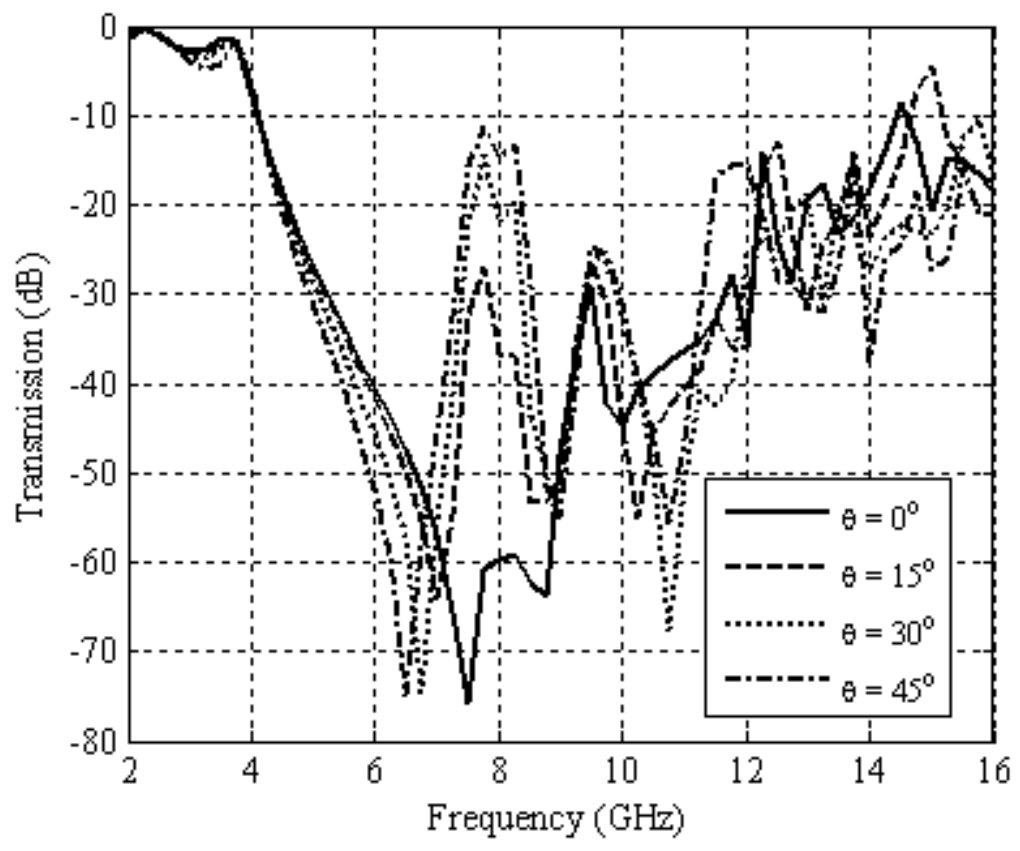

(a) Vertical polarization.

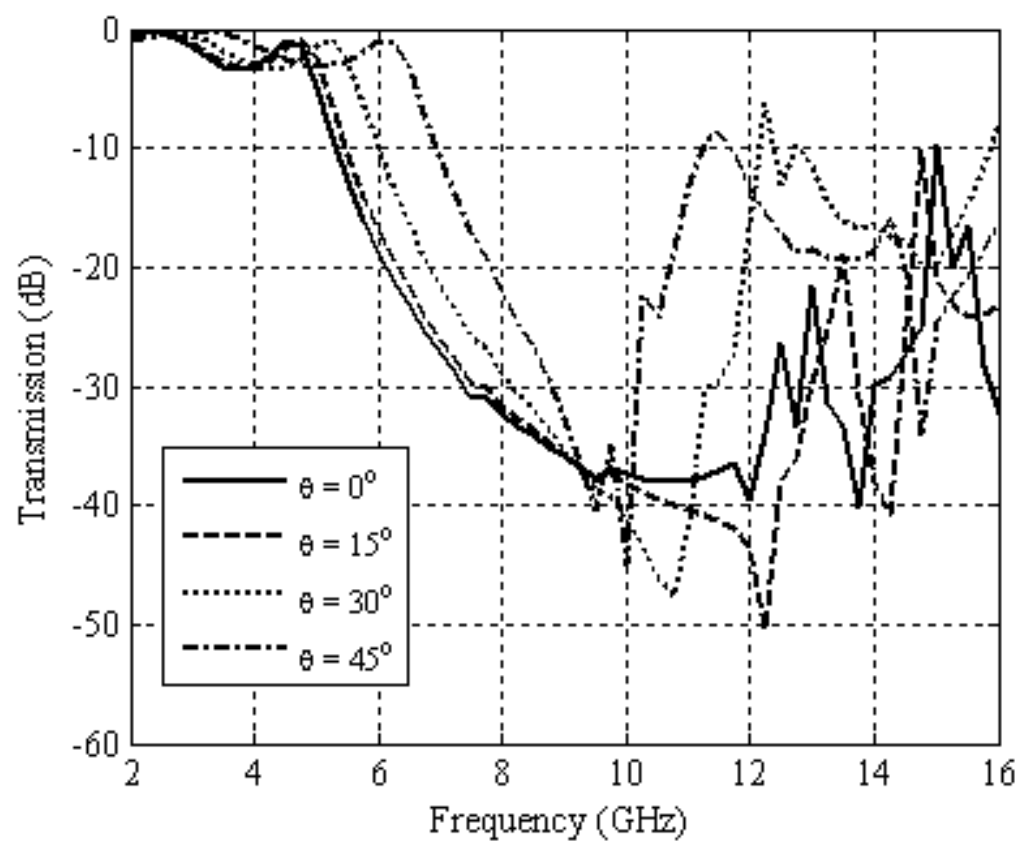

(b) Horizontal polarization.

Fig. 11. Transmission coefficient curves of FSS composite structure versus frequency for various angles of incidence. 


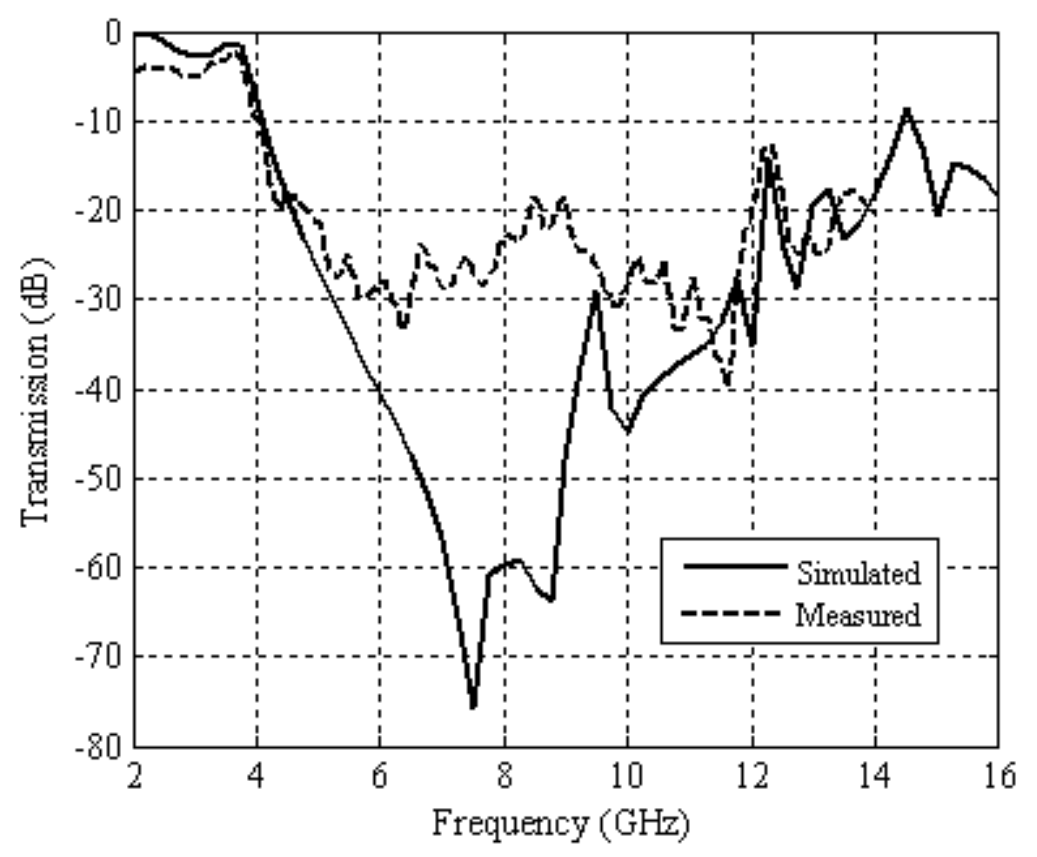

Fig. 12. Comparison between numerical and measured results for a three layer FSS for vertical polarization.

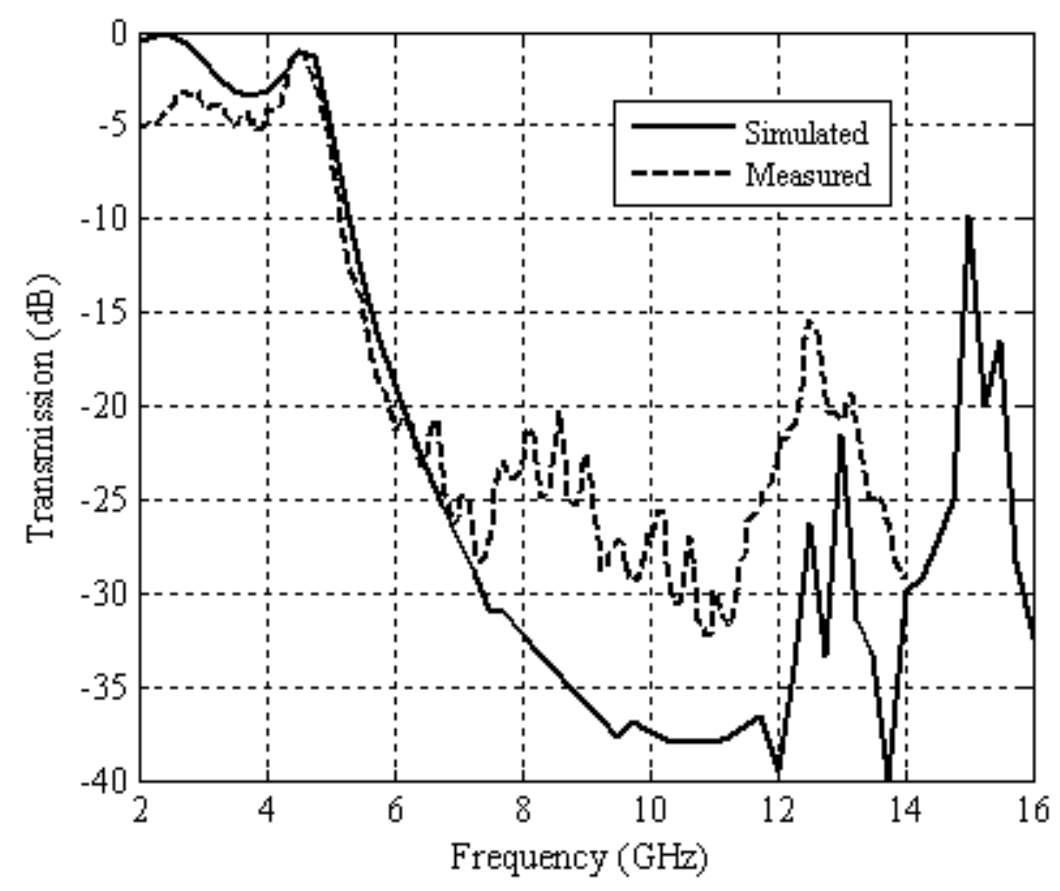

Fig. 13. Comparison between numerical and measured results for a three layer FSS for horizontal polarization.

Those results show that the structure can be used in UWB applications once that this technology has a bandwidth of $7.5 \mathrm{GHz}(3.10 \mathrm{GHz}-10.60 \mathrm{GHz})$ [11]. Measured results show that the bandwidth starts from $4.05 \mathrm{GHz}$, for vertical polarization, and for $5.05 \mathrm{GHz}$, for horizontal polarization, what is bigger than the lower frequency of UWB applications $(3.10 \mathrm{GHz})$. These differences may be adjusted with simple modifications in dimension of the unit cells. The higher frequency $(10.6 \mathrm{GHz})$ is into the 
bandwidth of the UWB FSS for both polarizations. The stop frequency was not detected in measured results due to limitations in the frequency range of the vector network analyzer used in measurements (10 MHz - $14 \mathrm{GHz}$ ). A little difference regards with bandwidth was obtained for vertical and horizontal polarization, what shows that the structure has polarization stability. This stability can be improved if equal periodicities were used in $x$ and $y$ directions.

Fig. 14 illustrates the phase of the reflection coefficient of the FSS $\left(S_{11}\right)$. It is evident that the remains almost constant oscillating in a mean value of 163 o over a stopband bandwidth. This shows that the FSS designed don't can be used in pulsed systems where linearly deceasing phase can be a key requirement.

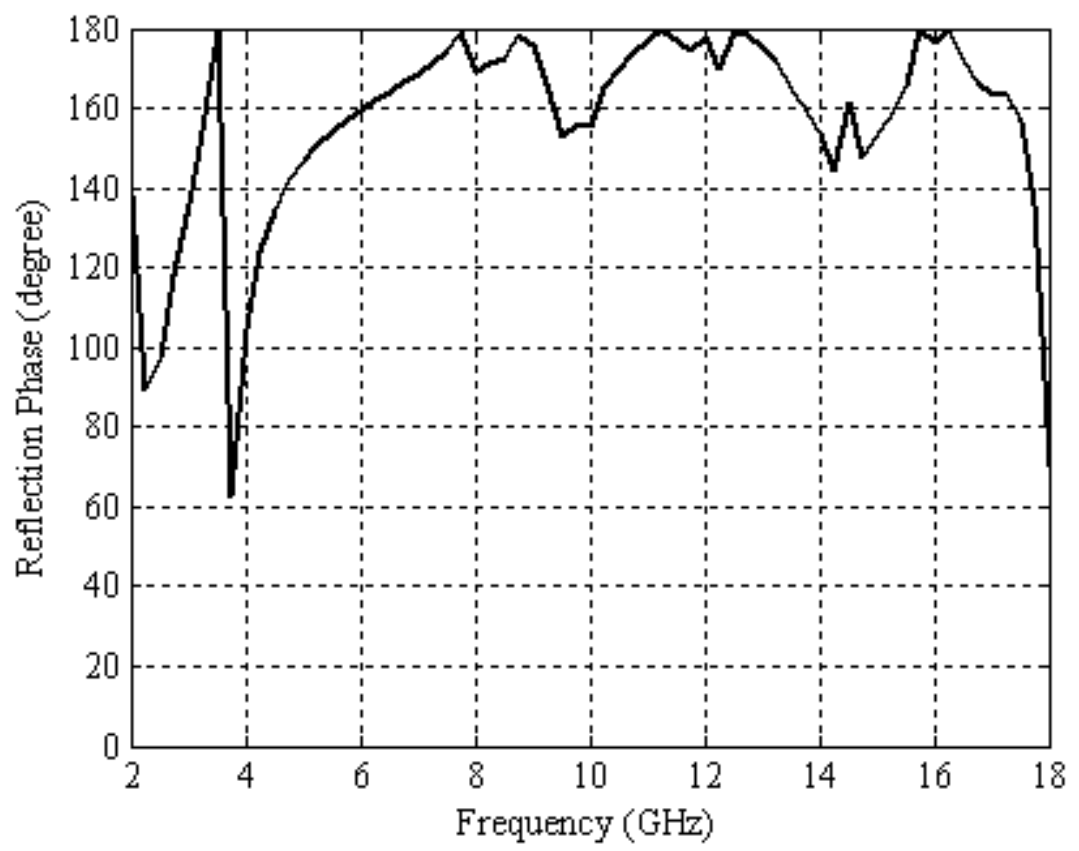

Fig. 14. The reflection phase coefficient.

\section{CONCLUSIONS}

This paper presented a new proposal for the design of a FSS bandstop filter with ultrawideband behavior. The structure was designed using three FSS layers with rectangular patch and rectangular patch with notches printed on a fiberglass dielectric substrate. A numerical analysis of transmission properties of the designed individuals FSS was performed through simulations using Ansoft DesignerTM commercial software. A numerical analysis of transmission properties of the designed UWB FSS was performed. The proposed FSS proves to be a potential candidate for UWB applications.

\section{REFERENCES}

[1] L. M. Araújo, R. H. C. Maniçoba, A. L. P. S. Campos, and A. G. d'Assunção, "A Simple Dual-Band Frequency Selective Surface”, Microwave and Optical Technology Letters, Vol. 51, No. 4, pp. 942 - 944, 2009.

[2] A. Kazemzadeh and A. Karlsson, "Multilayered Wideband Absorbers for Oblique Angle of Incidence", IEEE Transactions on Antennas and Propagation, Vol. 58, No. 11, pp. 3637 - 3646, 2010.

[3] Y. Ranga et al., "Design and Analysis of Frequency-Selective Surfaces for Ultrawideband Applications", 2011 IEEE EUROCON - International Conference on Computer as a Tool, 2011. 
[4] Y. Ranga et al., "Multilayer Frequency-Selective-Surface Reflector for Constant Gain over Ultra Wideband", Proceedings of the 5th European Conference on Antennas and Propagation (EUCAP), pp. 332 - 334, 2011.

[5] M. Pasian et al., "Frequency Selective Surfaces for Extended Bandwidth Backing Reflector Functions", IEEE Transactions on Antennas and Propagation, Vol. 58, No. 1, pp. 43 - 50, 2010.

[6] S. Baisakhiya et al., Novel Compact UWB Frequency Selective Surface For Angular And Polarization Independent Operation, Progress in Electromagnetics Research Letters, Vol. 40, pp. 71 - 79, 2013.

[7] W. Li et al., "Novel Frequency Selective Surfaces with compact structure and ultra-wideband response", 2012 AsiaPacific Symposium on Electromagnetic Compatibility (APEMC), pp. 557 - 560, 2012.

[8] R. M. S. Cruz, A. G. d'Assunção, and P. H. F. Silva, "A new FSS design proposal for UWB applications", 2010 International Workshop on Antenna Technology (iWAT), pp. 1-4, 2010.

[9] I. Sohail, Y. Ranga; K. P. Esselle; and S. G. Hay, "A frequency selective surface with a very wide stop band", 2013 7th European Conference on Antennas and Propagation (EuCAP), Gothenburg, Sweden, 2013.

[10] A. N. Silva, F. M. Pontes, L. S. de Oliveira, J. C. Silva, and A. Gomes Neto, "Frequency Selective Surfaces With non Fractal Geometry", Proceedings of the 2011 SBMO/IEEE MTT-S International Microwave and Optoelectronics Conference, 2011.

[11] M. Lad, "Ultra-Wideband: The Next Generation Personal Area Network Technology”, White Paper, 2004. 